\title{
Comparative effects of mesenchymal progenitor cells, endothelial progenitor cells, or their combination on myocardial infarct regeneration and cardiac function
}

\author{
Erik J. Suuronen, PhD, ${ }^{\mathrm{a}, \mathrm{b}}$ Joel Price, MD, ${ }^{a}$ John P. Veinot, $M{ }^{,}{ }^{\mathrm{c}}$ Kathryn Ascah, MD, ${ }^{\mathrm{d}}$ Varun Kapila, MD, ${ }^{\mathrm{a}}$
} Xue-Wei Guo, MD, PhD, ${ }^{a}$ Serena Wong, MSc, ${ }^{a, b}$ Thierry G. Mesana, MD, PhD, ${ }^{a}$ and Marc Ruel, MD, MPH ${ }^{a, b}$

From the Divisions of Cardiac Surgery and Cardiology, ${ }^{\mathrm{d}}$ and Departments of Cellular and Molecular Medicine ${ }^{\mathrm{b}}$ and Pathology and Laboratory Medicine, ${ }^{\mathrm{c}}$ University of Ottawa Heart Institute, Ottawa, Ontario, Canada.

Supported by award 7346 from the Canadian Foundation for Innovation (to Dr Ruel), by grant MOP-77536 from the Canadian Institutes of Health Research (to Drs Ruel and Suuronen), and by a Heart and Stroke Foundation of Canada/AstraZeneca Canada, Inc, Fellowship (to Dr Suuronen).

Received for publication March 6, 2007; revisions received June 29, 2007; accepted for publication July 16, 2007.

Address for reprints: Marc Ruel, MD, MPH, University of Ottawa Heart Institute, Division of Cardiac Surgery, 40 Ruskin St, Laboratory H553, Ottawa, Ontario, Canada, K1Y 4W7 (E-mail: mruel@ottawaheart.ca).

J Thorac Cardiovasc Surg 2007;134:1249-58 $0022-5223 / \$ 32.00$

Copyright (c) 2007 by The American Association for Thoracic Surgery

doi:10.1016/j.jtcvs.2007.07.028
Objective: Recent evidence suggests that the effects of mesenchymal progenitor cell transplantation into the infarcted myocardium might be mediated by local paracrine angiogenesis. We compared the effects of mesenchymal progenitor cell transplantation versus those of a primarily angiogenic cell, the endothelial progenitor cell, in a rat model of myocardial infarction.

Methods: Twenty-one days after left anterior descending artery ligation, rats were injected in their infarcted anterior myocardium with $1 \times 10^{6}$ mesenchymal progenitor cells, $1 \times 10^{6}$ endothelial progenitor cells, $5 \times 10^{5}$ mesenchymal progenitor cells plus $5 \times 10^{5}$ endothelial progenitor cells, or phosphate-buffered saline $(\mathrm{n}=$ 6-8 per group). Echocardiography was performed before injection and 4 weeks later, after which rats were killed and immunohistochemical analyses performed.

Results: Connexin 43 density was greater in cell-treated groups compared with that seen in the phosphate-buffered saline group (by $91.6 \% \pm 15.2 \%, P<.001$ ), with no observed difference between cell-treated groups $(P \geq .3)$. Endothelial progenitor cell treatment increased arteriolar density within the infarct border zone (by 297\%, $205 \%$, and $101 \%$ vs phosphate-buffered saline, mesenchymal progenitor cell, and mesenchymal progenitor cell/endothelial progenitor cell treatment, respectively; $P<.01$ ). Postoperative left ventricular ejection fraction (endothelial progenitor cell: $68.3 \% \pm 9.8 \%$ vs mesenchymal progenitor cell/endothelial progenitor cell: $55.0 \% \pm$ $11.1 \%$, mesenchymal progenitor cell: $53.0 \% \pm 6.0 \%$, and phosphate-buffered saline: $49.6 \% \pm 9.5 \%$ ) and fractional shortening (endothelial progenitor cell: $32.4 \% \pm$ $5.1 \%$ vs mesenchymal progenitor cell: $22.5 \% \pm 5.4 \%$ and phosphate-buffered saline: $21.3 \% \pm 5.3 \%$ ) were greater in endothelial progenitor cell-treated rats versus those receiving other treatments (all $P<.05$ ). Only endothelial progenitor cells prevented further contractile deterioration compared with baseline values $(P=$ .8 ), whereas other groups had continued loss of function after treatment.

Conclusion: Compared with the use of mesenchymal progenitor cells, cell transplantation with endothelial progenitor cells after myocardial infarction resulted in better neovascularization and contractility. This suggests that angiogenesis is an important mechanism in attenuating the progression of left ventricular dysfunction after myocardial infarction.

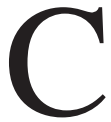

ell-based myocardial regenerative therapies aim at safely using bone marrow-, blood-, or tissue-derived progenitor cells to restore perfusion and function to chronically ischemic, stunned, hibernating, or scarred myocardial areas and at improving patient quality of life and survival beyond the effects of other available therapeutic modalities. To date, this remains an elusive goal.

Under selected experimental conditions, bone marrow-derived mesenchymal progenitor cells (MPCs) have been reported to generate cardiomyocytes ${ }^{1,2}$ and 


\author{
Abbreviations and Acronyms \\ EPC = endothelial progenitor cell \\ FS = fractional shortening \\ HPS = hematoxylin-phloxine-saffron \\ $\mathrm{LV}=$ left ventricular \\ $\mathrm{LVEF}=$ left ventricular ejection fraction \\ LVID $=$ left ventricular internal dimension \\ MI = myocardial infarction \\ MPC = mesenchymal progenitor cell \\ PBS = phosphate-buffered saline
}

therefore constitute a candidate for cell-based therapy. In myocardial infarction (MI) models, transplantation of MPCs into the infarct region has been shown to improve left ventricular ejection fraction (LVEF) and cardiac function. ${ }^{3}$ However, studies on large animals and early clinical investigations of MPC therapy after MI have showed only modest regional contractile improvements with little or no global recovery, and underlying mechanisms remain unclear. ${ }^{4-6}$ Recent evidence suggests that MPCs do not result in the formation of functional syncytia and that their effects in post-MI scarring might be mediated more by local paracrine angiogenesis than by cardiomyocyte differentiation. ${ }^{3,7}$ Hence, angiogenesis (the formation of new blood vessels) might constitute an important mechanism of functional improvement not only among modalities that specifically aim at angiogenesis (ie, for the treatment of myocardial ischemia in the presence of viable heart muscle), but also among modalities aiming at myogenesis to improve cardiac function after an MI (ie, for the treatment of heart failure in the presence of nonviable myocardium). ${ }^{8,9}$

If angiogenesis is the underlying mechanism for improved cardiac function after MPC treatment after MI, then transplantation of angiogenic/vasculogenic progenitors might constitute another cell-based approach to achieve post-MI recovery. Among the potential sources of stem/progenitor cells, circulating endothelial progenitor cells (EPCs) and their derivatives have important angiogenic properties. ${ }^{10}$ EPCs can be recruited from the blood to sites of angiogenesis, differentiate into endothelial cells, and proliferate to form new vasculature ${ }^{11}$ and might provide mitogenic factors for mature endothelial cells. ${ }^{12}$ Animal models have demonstrated a role for EPCs in enhancing vascularization in the infarcted, as well as in the ischemic, heart. ${ }^{13,14}$

Two clinical trials have examined the effects of intracoronary marrow-derived cells versus EPC injections for the treatment of MI, without any observed difference in recovery between the 2 cell-treated groups. ${ }^{5,15}$ However, no study to date has compared MPCs versus EPCs by using intramyocardial cell delivery, which is more targeted and effective than intracoronary injection. ${ }^{16}$ Furthermore, no study has directly compared the underlying mechanisms of recovery and regeneration after cell therapy by using these 2 widely used cell populations. Therefore we compared and combined the use of MPCs and circulatory EPCs for cellbased therapy in a rat MI model and examined the hypothesis that the EPC, because of its high angiogenicity, ${ }^{10,11}$ might elicit an equal or better therapeutic response than the MPC. The data presented herein suggest that focusing on the transplantation of primarily vasculogenic cells, such as the EPC, might be an equally viable or even superior cell-based approach for the treatment of post-MI cardiac dysfunction.

\section{Materials and Methods \\ Cell Isolation and Culture}

MPCs and EPCs were isolated from healthy donor syngeneic Sprague-Dawley rats (Charles River, Wilmington, Mass) weighing 200 to $250 \mathrm{~g}$ that did not undergo MI induction or any other manipulation. For EPCs, blood was collected from the aortas of anesthetized rats ( $2 \%$ isoflurane). Total peripheral blood mononuclear cells were isolated and cultured, as described previously. ${ }^{17}$ Briefly, cultures were supplemented with endothelial basal medium with EGM-2-MV-SingleQuots (Clonetics, Guelph, Canada), and day 4 adherent cells represented the EPC population. Characteristic of EPCs, these cells stained positive for 1,1'-dioctadecyl3,3,3',3'-tetramethylindocarbocyanine-labeled acetylated lowdensity lipoprotein uptake and fluorescein isothiocyanate-labeled Ulex europaeus agglutinin 1 binding (not shown). For MPCs, tibias and femurs were dissected from rats after death. Bone marrow was extruded from rat tibias and femurs by using a needle and syringe and flushing with phosphate-buffered saline (PBS). Cells were isolated from the bone marrow extract by means of collagenase treatment ( $250 \mu \mathrm{L} / \mathrm{mL}$; Sigma, Oakville, Canada) and then cultured at $1.3 \times 10^{5} \mathrm{cells} / \mathrm{cm}^{2}$ until confluent (approximately 14 days) in Dulbecco's modified Eagle's medium (Sigma) supplemented with $10 \%$ fetal bovine serum, L-glutamine $(2 \mathrm{mmol} / \mathrm{L})$, penicillin $(100 \mathrm{U} / \mathrm{mL})$, and streptomycin $(100 \mathrm{mg} / \mathrm{mL})$. Nonadherent erythroid progenitor cells were removed with each medium change, yielding a population of adherent MPCs that characteristically expressed the antigens CD29 and CD44 but were negative for the hemopoietic cell marker CD45. ${ }^{18}$ Before transplantation, MPCs or EPCs for single cell-type injections were labeled with $4^{\prime}, 6$-diamidino-2' -phenylindole (Sigma); in the case of dual celltype injections, MPCs were labeled as above, and EPCs were labeled with carboxyfluorescein diacetate (Molecular Probes, Eugene, Ore), according to the manufacturer's protocol.

\section{Animal Model}

Animal procedures (Figure 1) were performed with the approval of the University of Ottawa Animal Care Committee in accordance with the National Institute of Health's "Guide for the care and use of laboratory animals." Syngeneic Sprague-Dawley rats (200-250 g) were used for the infarction and cell transplantation model. Left coronary artery ligation procedures were performed by Charles River surgical services, and animals were shipped after recovery.

Baseline echocardiography was performed 14 days after ligation (as below). Animals were then randomized to receive injec- 

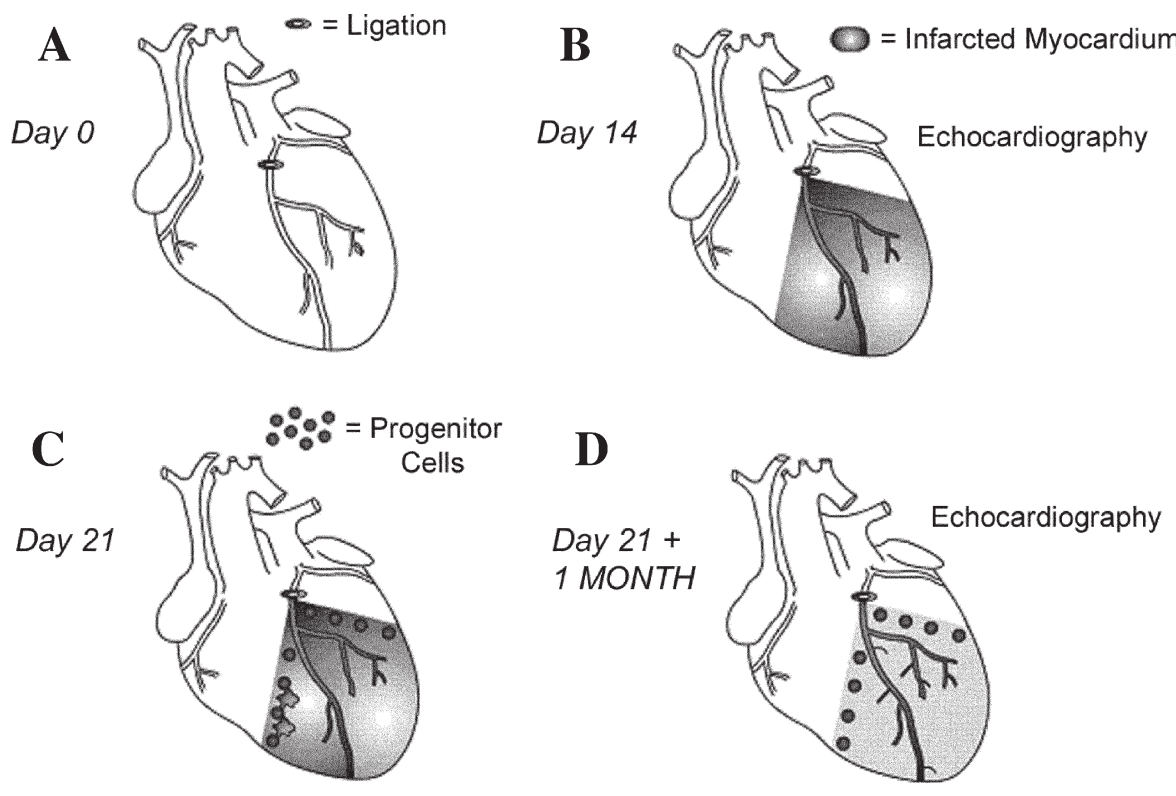

Figure 1. Experimental design. A, The left descending coronary artery was ligated. B, Echocardiographic analysis was performed 14 days after ligation. C, At post-myocardial infarction day 21, cell transplantation was performed into the infarcted region. D, Echocardiography was performed 1 month after cell transplantation, and the experiment was terminated. tions of cells or PBS (controls) 7 days later. The lack of difference in baseline ejection fraction and fractional shortening (FS) values between groups confirmed an equivalent degree of infarction between treatment groups. Mechanical ventilation was established in anesthetized rats (isoflurane), and the heart was exposed through a left thoracotomy. By using a 27-gauge needle, 5 equivolumetric intramyocardial injections $(200 \mu \mathrm{L}$ in total) were performed in a standardized fashion, one at each border and one in the middle of the dyskinetic and discolored anterior myocardial area representing the infarction. Randomized animals were injected with one of the following in PBS: (1) $1 \times 10^{6}$ MPCs; (2) $1 \times 10^{6}$ EPCs; (3) $5 \times 10^{5}$ MPCs plus $5 \times 10^{5}$ EPCs; or (4) PBS only.

\section{Echocardiographic Analysis}

Baseline (14 days after MI and 7 days before cell transplantation) and posttreatment (after 28 days) transthoracic echocardiographic analysis of rat hearts were performed by using a small animalcompatible analog echocardiography system (Philips, Markham, Canada). Rats were anesthetized (isoflurane) in the supine position, and their chests were shaved. The heart was imaged in a short-axis view of the left ventricle at the level of the papillary muscle. End-diastolic and end-systolic left ventricular (LV) internal dimension (LVID) measurements were taken. LVEF, FS, and the end-diastolic and end-systolic LV volume indices (in microliters per gram of body weight) were calculated. Measurements were interpreted by 2 blinded echocardiographers and averaged. Reported values have been normalized to baseline values for all treatment groups.

\section{Histologic Assessment}

After death, hearts were immediately dissected and fixed with $4 \%$ paraformaldehyde. Tissue was then stored in $10 \%$ neutral-buffered formalin, serially sectioned, and paraffin embedded. Slides were prepared by using $4-\mu \mathrm{m}$ serial sections at different levels. Sections were stained with hematoxylin-phloxine-saffron (HPS) stain- ing. For determining infarct size and length and for measuring the anterior and posterior LV wall thickness, computer-assisted planimetry was performed. Infarct size was calculated as the infarcted area divided by the total LV area. The inflammatory cell reaction was estimated by grading the number of leukocytes per HPS-stained tissue section (0, none; 1, low; 3, medium; 5, severe). Vascular density in the scar and border zone was calculated as the number of arterioles per square millimeter by using HPS-stained slides and slides stained for $\alpha$-smooth muscle actin ( $\alpha$-SMA; see below). Counts were determined from 6 random microscopic fields (at a minimum of 3 tissue levels) per sample in a blinded fashion.

Cardiac troponin T, $\alpha$-SMA, and connexin 43 were localized by means of immunohistochemical staining with anti-cardiac troponin T (1:50; Santa Cruz Biotechnology, Santa Cruz, Calif), anti- $\alpha$ SMA (1:400; Chemicon, Temecula, Calif), and anti-connexin43 (1:200; Chemicon) antibodies, according to the manufacturer's protocol. Differentiation of transplanted cells into cardiomyocytes was assessed on the basis of the expression of cardiac troponin T. The density of $\alpha$-SMA-expressing myofibroblasts was calculated and reported as the area of $\alpha$-SMA-positive staining/ infarct area (as a percentage), while excluding characteristic $\alpha$-SMA-positive staining of arteriolar vessel walls. The $\alpha$-SMA staining also identified smooth muscle cells in arterioles, which were distinguished from myofibroblasts by their distinctive morphology. The densities of intercellular connexin43 staining were measured in infarct and peri-infarct area myocytes and reported as the number of intercalated discs that contained connexin43/myocyte. All counts were determined from 6 random microscopic fields (at a minimum of 3 tissue levels) per sample in a blinded fashion.

\section{Statistics}

Values are expressed as means \pm standard deviation of the mean. Statistical analyses were performed with Intercooled Stata 8 soft- 

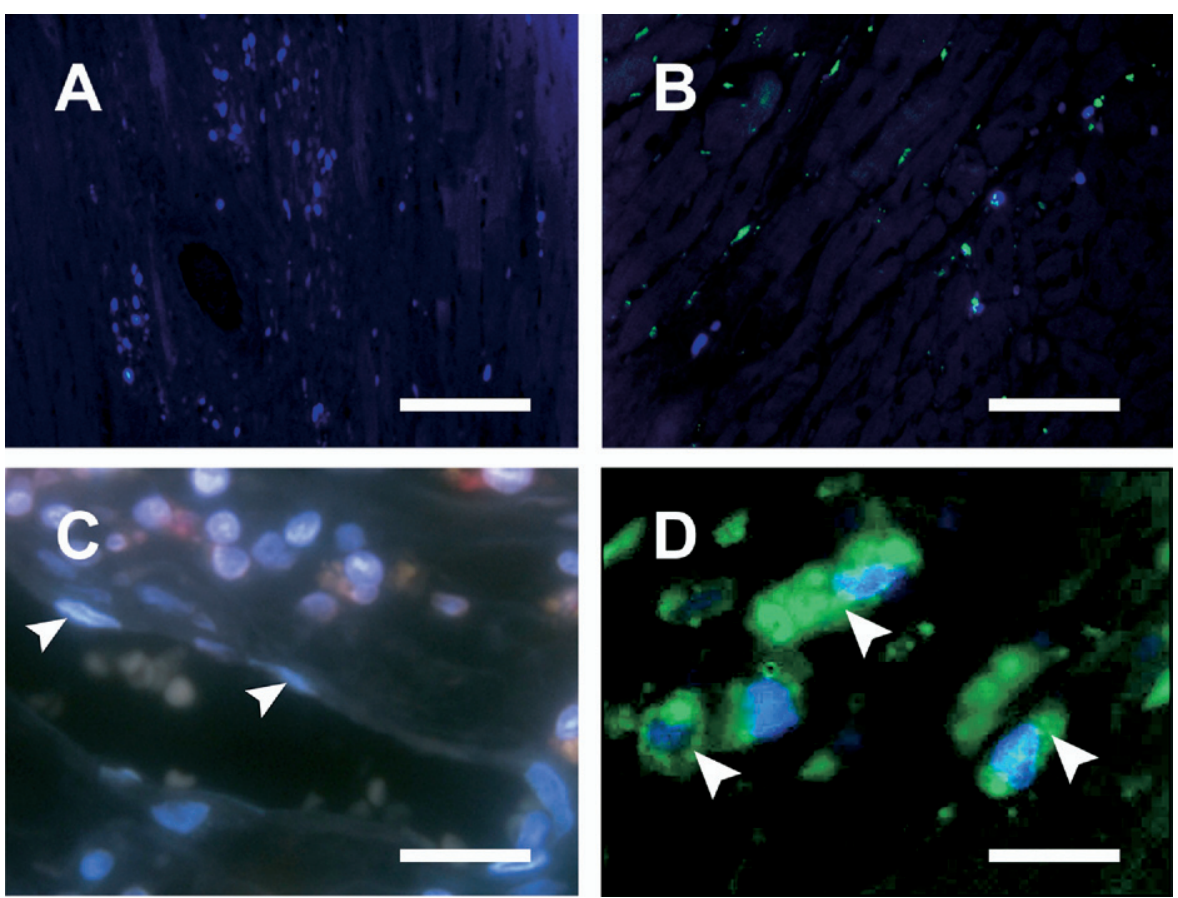

Figure 2. Cell engraftment and differentiation. Representative images of the following are shown: A, 4',6-diamidino2 ' -phenylindole (DAPI)-labeled nuclei (blue) of mesenchymal progenitor cells (MPCs) within the scar tissue; B, cotransplanted endothelial progenitor cells (EPCs; green) and MPCs (b/ue) in the border zone; C, evidence for endothelial differentiation of engrafted DAPI-positive EPCs (arrowheads) within vessels in the infarct; and D, evidence for cardiomyocyte differentiation of engrafted DAPIpositive MPCs (arrowheads) with coexpression of cardiac troponin $\mathrm{T}$ (green). Scale bar $=250 \mu \mathrm{m}$ (A and B), $40 \mu \mathrm{m}$ (C), and $30 \mu \mathrm{m}$ (D). ware (StataCorp, College Station, Tex). Comparisons of continuous data between groups were performed with a 1-way analysis of variance adjusted for repeat measures, a 2-tailed Student $t$ test, or both, using Bonferroni corrections as appropriate.

\section{Results}

Three (9\%) of 32 rats died after coronary ligation and before injection, and a further loss of 2 animals occurred after cell treatment. These animals were excluded from the analyses. The final sample sizes per treatment group were as follows: (1) MPCs $(\mathrm{n}=6)$; (2) EPCs $(\mathrm{n}=7)$; (3) MPCs/EPCs $(\mathrm{n}=$ $8)$; and (4) PBS $(\mathrm{n}=6)$.

Engraftment and Differentiation of Transplanted Cells After 4 weeks, the majority of EPC and MPC engraftment occurred within the scar regions and border zone (Figure 2, $A$ and $B$ ); however, some labeled cells were also observed within the healthy myocardium. EPCs were observed to incorporate into the endothelial walls of blood vessels (Figure 2,C), whereas MPCs were not. Only transplanted MPCs were found to express the cardiomyocyte marker troponin $\mathrm{T}$ (Figure 2,D); however, expression was observed only in 7 of 102 sections examined.

\section{Histologic Assessment of Infarcts}

Pathologic assessment revealed no significant difference in transmurality or inflammation $(P=.52$ and .25 , respectively; data not shown) between the treatment groups. The size of the infarcted myocardium was similar between treatments (Table 1). The EPC-treated group demonstrated re- duced infarct length and greater anterior/posterior wall thickness ratio (Table 1$)$. Less myofibroblasts $(0.25 \% \pm$ $0.11 \%$ ) were observed in the EPC-treated group compared with the PBS-, MPC-, and MPC/EPC-treated groups $(2.59 \% \pm$ $0.58 \%, 1.09 \% \pm 0.32 \%$, and $0.63 \% \pm 0.17 \%$, respectively; Figure 3). The potential for intercellular communication between cardiomyocytes was assessed on the basis of intercardiomyocyte connexin43 density (Figure 4, $A$ and $B$ ). Connexin43 staining was greater in cell-treated groups compared with that seen in the PBS-treated group (by $91.6 \% \pm$ $15.2 \%$ ), but no difference was observed among cell groups (Figure 4,C).

EPC-treated hearts demonstrated greater vascular density within the scar territory. EPC treatment alone resulted in significantly greater arteriolar density within the infarct $\left(23.3 \pm 13.1\right.$ arterioles $\left./ \mathrm{mm}^{2}\right)$ compared with PBS, MPC alone, and MPC/EPC treatment $(6.7 \pm 4.3,9.9 \pm 4.6$, and $12.1 \pm 5.3$ arterioles $/ \mathrm{mm}^{2}$, respectively). This represents increases in arteriolar density of $248 \%, 135 \%$, and $93 \%$ after EPC treatment versus PBS, MPC, and MPC/EPC treatment. More notably, arteriole density was significantly greater in the infarct border zone of EPC-treated hearts $\left(62.3 \pm 15.5\right.$ arterioles $\left./ \mathrm{mm}^{2}\right)$ versus those undergoing PBS, MPC, and MPC/EPC treatment (15.7 $\pm 3.6,20.4 \pm 3.9$, and $31.0 \pm 9.0$ arterioles $/ \mathrm{mm}^{2}$, respectively; Figure 5, A-D). This represents increases in arteriolar density of $297 \%$, $205 \%$, and $101 \%$ after EPC treatment versus that after PBS, $\mathrm{MPC}$, and MPC/EPC treatment. The results for vessel densities are summarized in Figure 5, E. 
TABLE 1. Histologic and echocardiographic infarct parameters at 28 days after treatment

\begin{tabular}{|c|c|c|c|c|}
\hline & PBS & MPC & EPC & MPC/EPC \\
\hline \multicolumn{5}{|l|}{ Histology-planimetry } \\
\hline Infarcted area & $0.37 \pm 0.08$ & $0.36 \pm 0.05$ & $0.35 \pm 0.10$ & $0.37 \pm 0.07$ \\
\hline Infarct Length $(\mathrm{cm})$ & $1.17 \pm 0.10$ & $1.09 \pm 0.31$ & $0.82 \pm 0.15^{*}$ & $1.12 \pm 0.26$ \\
\hline AWT/PWT & $0.67 \pm 0.10$ & $0.76 \pm 0.15$ & $0.87 \pm 0.13^{*}$ & $0.71 \pm 0.14$ \\
\hline \multicolumn{5}{|l|}{ Echocardiographic analysis $†$} \\
\hline Rat weight $(\mathrm{g})$ & $343 \pm 18$ & $345 \pm 35$ & $340 \pm 19$ & $341 \pm 22$ \\
\hline Heart rate (beats/min) & $368 \pm 39$ & $392 \pm 43$ & $377 \pm 12$ & $365 \pm 29$ \\
\hline EDVI $(\mu \mathrm{L} / \mathrm{g})$ & $3.15 \pm 1.20$ & $2.12 \pm 0.62$ & $1.56 \pm 0.80^{*}$ & $2.77 \pm 1.57$ \\
\hline ESVI $(\mu \mathrm{L} / \mathrm{g})$ & $2.80 \pm 1.44$ & $0.95 \pm 0.36^{*}$ & $0.53 \pm 0.38^{*}$ & $1.38 \pm 1.00$ \\
\hline
\end{tabular}

Infarct size is shown as the ratio of infarcted area to total left ventricular area. Left ventricular wall thickness is calculated as the ratio of anterior wall thickness (AWT) to posterior wall thickness (PWT). End-diastolic (EDVI) and end-systolic (ESVI) left ventricular volume indices are normalized to body weight. $P B S$, Phosphate-buffered saline; $M P C$, mesenchymal progenitor cell; $E P C$, endothelial progenitor cell. * $P<.05$ versus phosphate-buffered saline treatment. †None of these parameters were significantly different at baseline.

\section{Function}

Animal heart rates were equal between groups (Table 1). As an indication of reduced dilatation, only EPC-treated rats had both lower end-diastolic and end-systolic LV volume indices (normalized for body weight, Table 1) compared with PBS control animals. The baseline LVEF, FS, and end-systolic and end-diastolic LVIDs were not significantly different between treatment groups (Figure 6). Four weeks after transplantation, LVEF decreased significantly from baseline in the PBS-treated $(69.0 \% \pm 11.7 \%$ to $49.6 \% \pm$ 9.5\%), MPC-treated $(68.3 \% \pm 18.9 \%$ to $53.0 \% \pm 6.0 \%)$, and MPC/EPC-treated $(69.4 \% \pm 7.4 \%$ to $55.0 \% \pm 11.1 \%$; Figure $6, A$ ) groups. In contrast, there was no significant change in LVEF for the EPC-treated group $(66.4 \% \pm$ $17.6 \%$ to $68.3 \% \pm 9.8 \%$; Figure $6, A$ ). Also, posttransplantation LVEF was greater in EPC-treated hearts compared with those undergoing all other treatments (by $\geq 24.1 \%$, $P<.05)$. After 28 days, FS was significantly greater in the EPC-treated hearts $(32.4 \% \pm 5.1 \%)$ compared with values in the other treatment groups $(21.3 \% \pm 5.4 \%$ and $22.6 \% \pm$ $5.6 \%$ for PBS and MPC treatment, respectively; Figure 6, $B)$. EPC treatment also prevented further changes in LVID measurements, with significantly reduced postoperative end-diastolic $(7.8 \pm 1.5 \mathrm{~mm})$ and end-systolic $(5.4 \pm 1.3$ $\mathrm{mm})$ LVID observed in EPC-treated hearts compared with that seen in PBS-treated control hearts $(10.1 \pm 1.4 \mathrm{~mm}$ and $9.6 \pm 2.0 \mathrm{~mm}$ for end-diastolic LVID and end-systolic LVID, respectively; Figure 6, $C$ and $D$ ).

\section{Discussion}

In this study, the vasculogenic, regenerative, and functional effects of EPCs and MPCs were directly compared in a rat MI model. It was demonstrated that (1) transplantation of EPCs was associated with a significant increase in vasculature within the MI and border zone, (2) EPCs but not MPCs were seen directly incorporating into endothelial walls of blood vessels, and (3) further loss of LV function after transplantation was prevented in the EPC-treated group only. This constitutes the first comparative study to demonstrate better preservation of myocardial function after MI using EPCs compared with that demonstrated using MPCs and to correlate this observation with superior neovascularization.

An important role for EPCs in angiogenesis has previously been established, ${ }^{11}$ and animal studies have demonstrated enhanced neovascularization with use of these cells in ischemic models. ${ }^{12,13}$ In this study, increased formation of capillaries was seen in infarcted myocardium when using EPCs compared with MPCs (data not shown). More importantly, EPC treatment resulted in greater numbers of fully formed arterioles in the border zone and infarct territories. Such vessels are presumably longer lasting than are the leaky small capillaries that result from local paracrine effects and inflammation. ${ }^{19}$ A lesser response was observed for the combined MPC/EPC treatment, possibly because of a dose effect, as demonstrated previously for the assessment of angiogenic therapy ${ }^{20}$ Overall, it is possible that EPCs might regenerate a more functional vasculature in the infarcted heart. This might be particularly important in the border zone, where stunned or hibernating myocardium (which is viable but with suboptimal function) could be recovered with the help of a restored blood supply, thus minimizing the degenerative process. ${ }^{21,22}$

Echocardiography was used to assess progression of LV dysfunction after cell transplantation with the various cell types. Contrary to the other treatment groups, when EPCs were transplanted alone, further loss in myocardial function (LVEF and FS) was prevented. These data suggest that enhanced angiogenesis after EPC injection might be associated with the prevention of further loss of myocardium (eg, stunned or hibernating), with the attenuation of adverse LV remodeling, or both. Mechanistically corroborating this statement is the observation that after 4 weeks (1) the level 

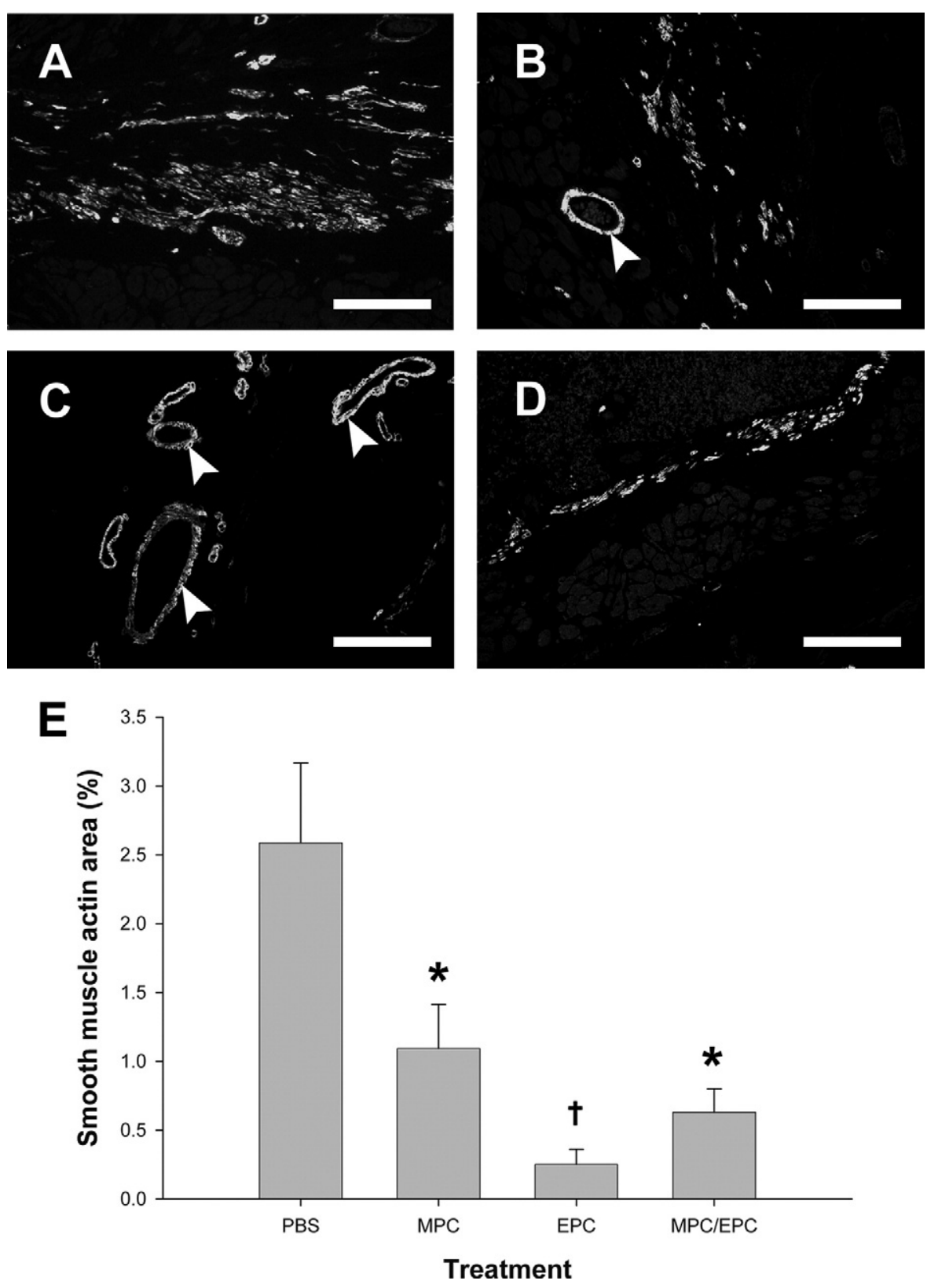

Figure 3. $\alpha$-Smooth muscle actin ( $\alpha$ SMA) staining. A-D, Representative images of $\alpha$-SMA staining in phosphatebuffered saline (PBS)-treated (A), mesenchymal progenitor cell (MPC) treated (B), endothelial progenitor cell (EPC)-treated (C), and MPC/EPCtreated (D) hearts. Arrows indicate positive $\alpha$-SMA staining in arterioles (note the many arterioles observed in EPC-treated hearts [C]), which was excluded from the calculation of myofibroblast density. E, The area of $\alpha$-SMA staining (percentage of total area) representing myofibroblast density for the 4 treatment groups after transplantation. ${ }^{*} \boldsymbol{P}<.05$ versus PBS; $\boldsymbol{t P}<.05$ versus all other treatments. Scale bar $=150 \mu \mathrm{m}$. of myofibroblasts, suggestive of adverse remodeling and scar formation, ${ }^{23}$ was lower in EPC-treated hearts compared with in hearts undergoing other treatments, and (2) only EPC treatment reduced infarct length and significantly prevented $\mathrm{LV}$ wall thinning and dilatation compared with that seen in PBS-injected control hearts. This is a novel finding in that previous trials comparing marrow-derived versus circulatory progenitor cells have been unable to demonstrate a difference in progression of $\mathrm{LV}$ dysfunction between cell types, ${ }^{5,15}$ but these trials did not use intramyocardial cell delivery, which constitutes a more specific delivery method. ${ }^{16}$ However, it is acknowledged that the marrow-derived cell populations used in the different trials and our studies are not identical (although their source of derivation is the same), possibly allowing for differential cell function on transplantation.

Although other studies have observed MPC differentiation into an endothelial phenotype within vessel walls of cell-implanted hearts, ${ }^{7}$ this study demonstrated EPCs, but not MPCs, incorporating into vascular structures. The differentiation potential of cells might be altered by differences in the severity of the pretreated infarct, the timing of cell transplantation, or both. The average baseline LVEF of animals in our study was $68 \%$, with cell injection performed 

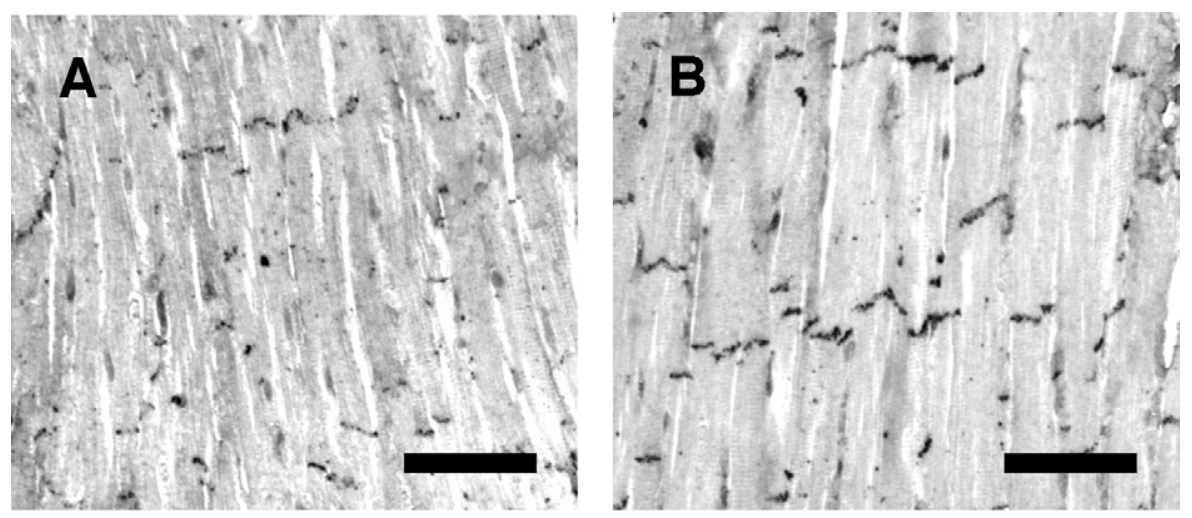

Figure 4. Connexin43 staining. A and B, Examples of connexin43 staining in border zone cardiomyocytes in phosphatebuffered saline (PBS)-treated (A) and endothelial progenitor cell (EPC)-treated (B) hearts. Scale bar $=50 \mu \mathrm{m}$. C, Intracellular connexin43 per myocyte in the infarct and peri-infarct areas for the 4 treatment groups. ${ }^{*} \boldsymbol{P}<.05$ versus the PBS group. MPC, Mesenchymal progenitor cell.

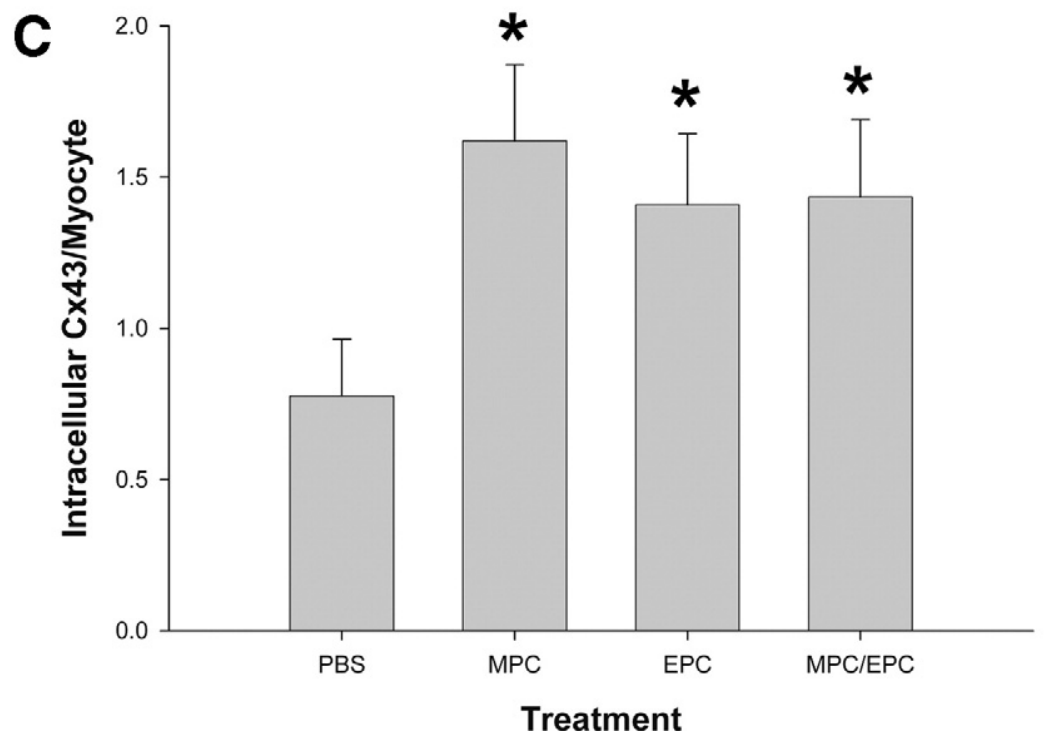

at 3 weeks after ligation. Many studies previously excluded animals with LVEFs of greater than $45 \%$, and consequently, the regenerating environments might have differently affected the milieu in which the transplanted cells differentiate. Although MPCs did not demonstrate endothelial differentiation, these cells were observed to express troponin $\mathrm{T}$, which is indicative of cardiomyocyte differentiation. However, the scope of this study did not assess the possibility that MPC expression of troponin $\mathrm{T}$ might have been the result of transdifferentiation or cell fusion, as previously reported. ${ }^{24}$ A limitation of the cell-labeling procedures used in this study is the possibility of false-positive identification from the loss of the label from the transplanted cells and uptake by host cells. However, this would not affect the vascular density or functional measurements, which were not dependent on cell identification but rather on the overall effect of the treatment.

As a consequence of the outlined cell-identification issues, a limitation of this study is that the level of engraft- ment of donor cells between treatments was not compared. It is possible that greater loss of one cell type versus the other was in part responsible for the observed differences in cardioprotective effect. It has been demonstrated previously that low cell survival can minimize the functional contribution of nonangiogenic cell populations. ${ }^{20,25} \mathrm{But}$ cell loss is a universal phenomenon of cell transplantation, ${ }^{25}$ and the numbers of cells transplanted in the present study were similar to that used in other EPC and MPC model studies. ${ }^{2,7,8}$ The cells in the present study were given equal opportunity to achieve a therapeutic response in equal environmental conditions. However, the environment might not be ideal for either cell type, and customized manipulation of the experimental conditions, such as angiogenic cotransfection or pretreatment, ${ }^{20,25}$ could improve the response to treatment with either cell type.

Similarly, improvements in functional parameters of the myocardium after various cell therapies have been widely 

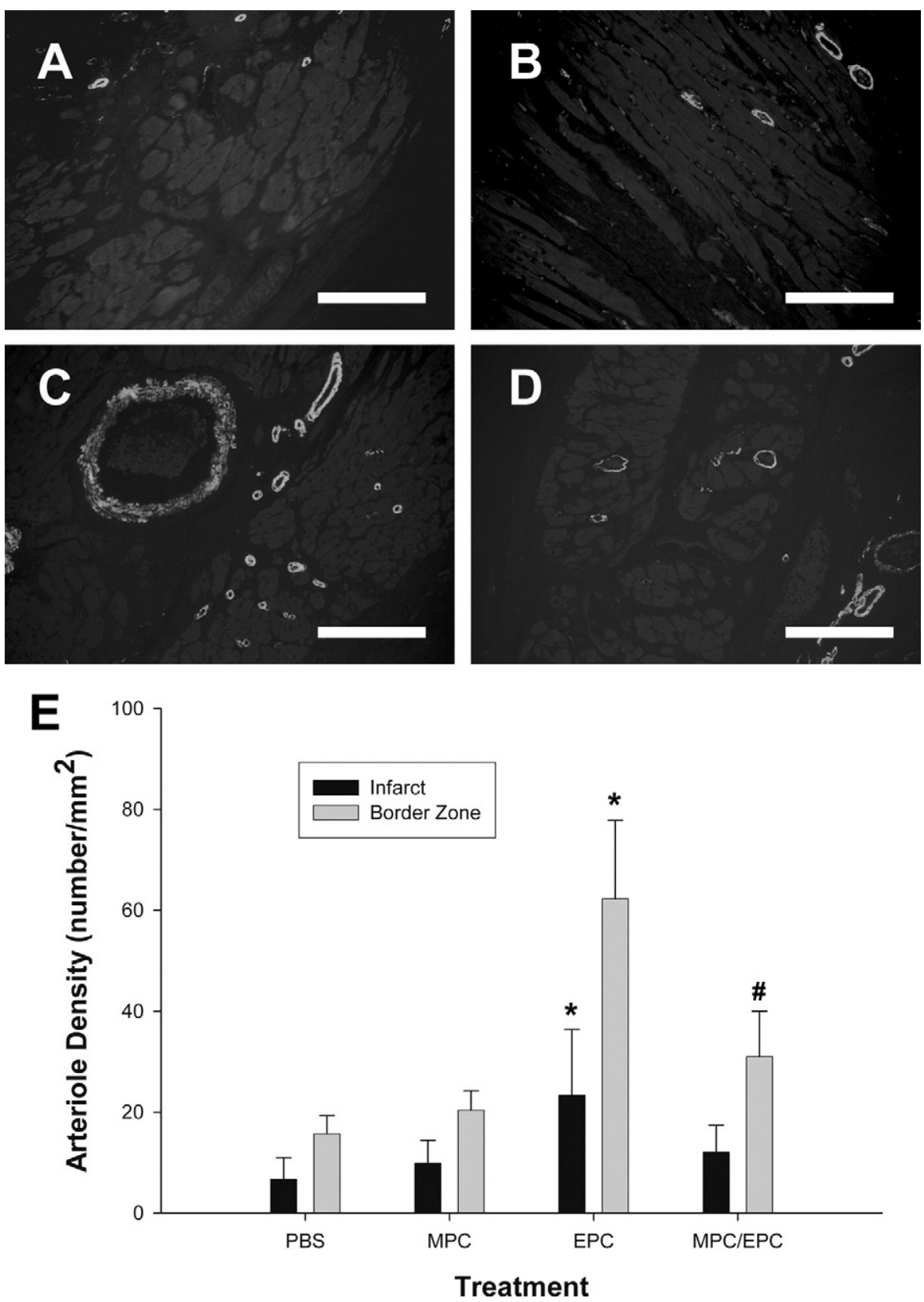

Figure 5. Vascular density. Representative $\alpha$-smooth muscle actin-stained sections of infarct border zone territories showing the positively stained smooth muscle cells of arterioles in hearts that received phosphate-buffered saline (PBS; A), mesenchymal progenitor cell (MPC; B), endothelial progenitor cell (EPC; C), or MPC/EPC (D) treatment. Scale bar = $200 \mu \mathrm{m}$. E, The number of arterioles (per square millimeter) in the infarct and border zone for the 4 treatment groups. ${ }^{*} \boldsymbol{P}<.01$ versus all other groups within territory (infarct or border zone); $\# \boldsymbol{P}<.05$ versus PBS-treated hearts. reported. ${ }^{26}$ In our infarct model, however, further deterioration was encountered in all except the EPC group. This adds to other recent evidence casting doubt on the true efficacy of MPC treatments in regenerating the myocardium. ${ }^{27}$ Furthermore, and as reviewed by Murry and colleagues, ${ }^{28}$ the benefit of a surprisingly wide range of cell types might result from improvement of the infarct's passive mechanical properties, amelioration of ventricular remodeling, and/or paracrine effects. In support of this, the present study demonstrated equivalent connexin43 density in cardiomyocytes of all cell-treated hearts, which was significantly greater than that of PBS-treated control hearts. Through a paracrine mechanism, the transplanted cells potentially increased connexin43 expression, as has been previously demonstrated. ${ }^{29}$

It might be premature to conclude that the EPC is a superior progenitor cell for cell-based myocardial restoration therapy. However, the present data demonstrated that transplanted EPCs enhance vasculogenesis compared with MPCs and that this was coupled with halted progression of LV dysfunction. This suggests that angiogenesis is an important mechanism for infarct functional recovery in cell-based therapy. Until myo- 

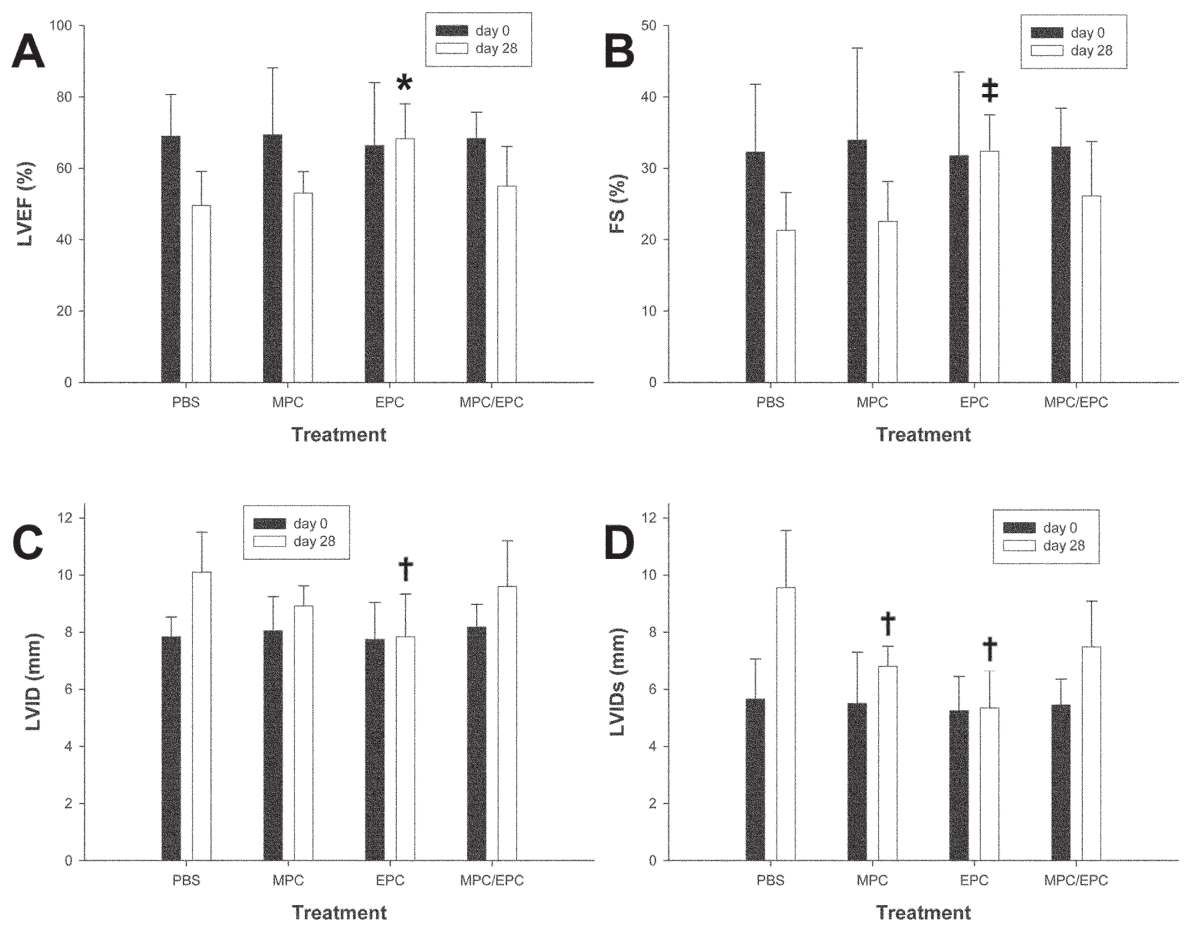

Figure 6. Assessment of cardiac function. Left ventricular ejection fraction ( $L V E F$; as a percentage; $A$ ); fractional shortening (FS; as a percentage; B), diastolic left ventricular internal dimension (LVID; in millimeters; C), and systolic LVID (in millimeters; D) at $\mathbf{0}$ and 28 days after treatment. ${ }^{*} \boldsymbol{P}<.05$ versus all other treatments at day 28; $\dagger P<.05$ versus phosphate-buffered saline (PBS) treatment at day $28 ; \mp P<$ .05 versus $P B S$ and mesenchymal progenitor cell (MPC) treatment at day 28. EPC, Endothelial progenitor cell. cardial syncytia-forming cell-based strategies are developed and functionally demonstrated, focusing on the transplantation of primarily vasculogenic cells, such as the EPC, might be an equally viable or even superior cell-based approach for the treatment of MI.

We thank Monir Ahmad, Stephanie Dean, Wendy PrichettPejic, and Rick Seymour for technical assistance.

\section{References}

1. Xaymardan M, Tang L, Zagreda L, Pallante B, Zheng J, Chazen JL, et al. Platelet-derived growth factor-AB promotes the generation of adult bone marrow-derived cardiac myocytes. Circ Res. 2004;94:e39-45.

2. Gojo S, Gojo N, Takeda Y, Mori T, Abe H, Kyo S, et al. In vivo cardiovasculogenesis by direct injection of isolated adult mesenchymal stem cells. Exp Cell Res. 2003;288:51-9.

3. Dai W, Hale SL, Martin BJ, Kuang JQ, Dow JS, Wold LE, et al. Allogeneic mesenchymal stem cell transplantation in postinfarcted rat myocardium: short- and long-term effects. Circulation. 2005;112: 214-23.

4. Janssens S, Dubois C, Bogaert J, Theunissen K, Deroose C, Desmet $\mathrm{W}$, et al. Autologous bone marrow-derived stem-cell transfer in patients with ST-segment elevation myocardial infarction: double-blind, randomised controlled trial. Lancet. 2006;367:113-21.

5. Schachinger V, Assmus B, Britten MB, Honold J, Lehmann R, Teupe $\mathrm{C}$, et al. Transplantation of progenitor cells and regeneration enhancement in acute myocardial infarction: final one-year results of the TOPCARE-AMI Trial. $J$ Am Coll Cardiol. 2004;44:1690-9.

6. Zhang M, Methot D, Poppa V, Fujio Y, Walsh K, Murry CE. Cardiomyocyte grafting for cardiac repair: graft cell death and anti-death strategies. J Mol Cell Cardiol. 2001;33:907-21.

7. Davani S, Marandin A, Mersin N, Royer B, Kantelip B, Herve P, et al. Mesenchymal progenitor cells differentiate into an endothelial phenotype, enhance vascular density, and improve heart function in a rat cellular cardiomyoplasty model. Circulation. 2003;108(suppl 1):II253-8.
8. Agbulut O, Vandervelde S, Al Attar N, Larghero J, Ghostine S, Leobon B, et al. Comparison of human skeletal myoblasts and bone marrow-derived $\mathrm{CD} 133+$ progenitors for the repair of infarcted myocardium. J Am Coll Cardiol. 2004;44:458-63.

9. Dimmeler S, Zeiher AM. Wanted! The best cell for cardiac regeneration. J Am Coll Cardiol. 2004;44:464-6.

10. Suuronen EJ, Wong S, Kapila V, Waghray G, Whitman SC, Mesana TG, et al. Generation of CD133(+) cells from CD133(-) peripheral blood mononuclear cells and their properties. Cardiovasc Res. 2006; 70:126-35.

11. Urbich C, Dimmeler S. Endothelial progenitor cells: characterization and role in vascular biology. Circ Res. 2004;95:343-53.

12. He T, Peterson TE, Katusic ZS. Paracrine mitogenic effect of human endothelial progenitor cells: role of interleukin-8. Am J Physiol Heart Circ Physiol. 2005;289:H968-72.

13. Asahara T, Kawamoto A. Endothelial progenitor cells for postnatal vasculogenesis. Am J Physiol Cell Physiol. 2004;287:C572-9.

14. Hristov M, Erl W, Weber PC. Endothelial progenitor cells: mobilization, differentiation, and homing. Arterioscler Thromb Vasc Biol. 2003;23:1185-9.

15. Dobert N, Britten M, Assmus B, Berner U, Menzel C, Lehmann R, et al. Transplantation of progenitor cells after reperfused acute myocardial infarction: evaluation of perfusion and myocardial viability with FDG-PET and thallium SPECT. Eur J Nucl Med Mol Imaging. 2004; 31:1146-51.

16. Hendrikx M, Hensen K, Clijsters C, Jongen H, Koninckx R, Bijnens E, et al. Recovery of regional but not global contractile function by the direct intramyocardial autologous bone marrow transplantation: results from a randomized controlled clinical trial. Circulation. 2006;114: I101-7.

17. Ruel M, Suuronen EJ, Song J, Kapila V, Gunning D, Waghray G, et al. Effects of off-pump versus on-pump coronary artery bypass grafting on function and viability of circulating endothelial progenitor cells. J Thorac Cardiovasc Surg. 2005;130:633-9.

18. Moadsiri A, Polchert D, Genrich K, Napoles P, Reina E, Turian J, et al. Mesenchymal stem cells enhance xenochimerism in NK-depleted hosts. Surgery. 2006;140:315-21. 
19. Nakamura Y, Yasuda T, Weisel RD, Li RK. Enhanced cell transplantation: preventing apoptosis increases cell survival and ventricular function. Am J Physiol Heart Circ Physiol. 2006;291:H939-47.

20. Baklanov D, Simons M. Arteriogenesis: lessons learned from clinical trials. Endothelium. 2003;10:217-23.

21. Beanlands RS, Hendry PJ, Masters RG, deKemp RA, Woodend K, Ruddy TD. Delay in revascularization is associated with increased mortality rate in patients with severe left ventricular dysfunction and viable myocardium on fluorine 18-fluorodeoxyglucose positron emission tomography imaging. Circulation. 1998;98(suppl):II51-6.

22. Haas F, Augustin N, Holper K, Wottke M, Haehnel C, Nekolla S, et al. Time course and extent of improvement of dysfunctioning myocardium in patients with coronary artery disease and severely depressed left ventricular function after revascularization: correlation with positron emission tomographic findings. J Am Coll Cardiol. 2000;36:1927-34.

23. See F, Thomas W, Way K, Tzanidis A, Kompa A, Lewis D, et al. p38 mitogen-activated protein kinase inhibition improves cardiac function and attenuates left ventricular remodeling following myocardial infarction in the rat. J Am Coll Cardiol. 2004;44:1679-89.
24. He XQ, Li SH, Weisel RD, Chen MS, Zhong Y, Lu WY, et al. Bone marrow mesenchymal stem cells acquire a cardiogenic phenotype via transdifferentiation and cell fusion with cardiomyocytes. Circulation. 2006;114(suppl):II412.

25. Retuerto MA, Schalch P, Patejunas G, Carbray J, Liu N, Esser K, et al. Angiogenic pretreatment improves the efficacy of cellular cardiomyoplasty performed with fetal cardiomyocyte implantation. J Thorac Cardiovasc Surg. 2004;127:1041-9.

26. Fazel S, Tang GH, Angoulvant D, Cimini M, Weisel RD, Li RK, et al. Current status of cellular therapy for ischemic heart disease. Ann Thorac Surg. 2005;79(suppl):S2238-47.

27. Deten A, Volz HC, Clamors S, Leiblein S, Briest W, Marx G, et al. Hematopoietic stem cells do not repair the infarcted mouse heart. Cardiovasc Res. 2005;65:52-63.

28. Murry CE, Reinecke H, Pabon LM. Regeneration gaps: observations on stem cells and cardiac repair. J Am Coll Cardiol. 2006;47:1777-85.

29. Kuhlmann MT, Kirchhof P, Klocke R, Hasib L, Stypmann J, Fabritz L, et al. G-CSF/SCF reduces inducible arrhythmias in the infarcted heart potentially via increased connexin43 expression and arteriogenesis. $J$ Exp Med. 2006;203:87-97. 